\title{
Decentralized Coordination of Autonomous Vehicles at intersections
}

\author{
L. Makarem*, D. Gillet* \\ * École Polytechnique Fédérale de Lausanne (EPFL), Switzerland
}

\begin{abstract}
In this paper, the decentralized coordination of point-mass autonomous vehicles at intersections using navigation functions is considered. As main contribution, the inertia of the vehicles is taken into account to enable on-board energy optimization for crossing. In such a way, heavier vehicles that need more energy and time for acceleration or breaking are given an indirect priority at intersections. The proposed decentralized coordination scheme of autonomous vehicles at intersection is compared with an optimal centralized coordination approach and traditional crossing of manned vehicles at intersection with traffic lights. The proposed decentralized coordination scheme shows a significant improvement in energy consumption and in motion smoothness compared to traditional crossing. It is also easier to deploy and more scalable than centralized approaches, with only a limited performance reduction.
\end{abstract}

Keywords: Autonomous Vehicles, Decentralized control, Decentralized Navigation Function

\section{INTRODUCTION}

Navigation of autonomous vehicles has been an attractive research area both in control and in robotics during the last decades. Accordingly, it could be expected that one day in the very near future autonomous or semi-autonomous driver assistance systems will be available to handle intersections. Autonomous navigation deals with the coordination of vehicles that carry out individual or collaborative tasks. It relies on the sharing and exploitation of information on the vehicles and their environment.

Different control approaches for autonomous navigation have been proposed in the literature. One approach is to describe the vehicles using kinematic models, with the objective of reaching a destination while avoiding collisions with fix and moving obstacles. In this approach, autonomous navigation is viewed as a multi objective problem for which various solutions have been suggested; such as stochastic optimization (Baras (2003)), cooperative methods of control (Roozbehani (2009.a)), and decentralized control (Roozbehani (2009.b)). Among all these methods, decentralized control has so far received more attention as a method that does not need long-range communication and shows more robustness to various communication failures in the system. The use of navigation functions in decentralized scheme seems promising, as it is feasible to be implemented in real-time and they also show good flexibility with regard to adding vehicles and changing environment (Roozbehani (2009a)) (Dimarogonas (2003)).

In this paper, the focus is on developing a flexible computationally efficient decentralized algorithm for vehicles at intersections while considering the different inertias of the vehicles and their priority for passing as well as their ability to break and accelerate at the intersections. As such, the vehicles need the ability to communicate and transfer data about their positions and their inertia. Our proposed navigation function is based on the distance of every vehicle with its destination (which can be a moving point) and with other vehicles and other obstacles. A zone of visibility is defined around each vehicle in order to emulate a real detection and communication range. Intersections are considered, as they correspond to traffic conditions having potentially a high impact on energy consumption and motion smoothness.

In section 2, a dynamical model of the vehicles is introduced. It is simple enough to enable the handling of complex traffic situations, and complex enough to enable energy optimization. The chosen intersection scenario is also detailed. In section 3, a decentralized navigation function that enables to take dynamical constraints into account is proposed. . This extended navigation function is analyzed in section 4 to make sure no deadlock situations are induced. The evaluation of the proposed approach is presented in section 5. It is compared with an optimal centralized coordination approach and traditional crossing of manned vehicles at intersection with traffic lights Finally, concluding remarks and outlook are given in section 6 .

\section{PROBLEM FORMULATION}

The considered multi-vehicle system consists of $N$ vehicles. These vehicles have been modeled using second order dynamics:

$\ddot{q}_{i}=\frac{1}{m_{i}} u_{i}$

Where $q_{i}$ and $u_{i}$ are the state variable and control input of the vehicle $i$ respectively. $q_{i}$ is the position of the vehicle $i$. $\dot{q}_{i}$ and $\ddot{q}_{i}$ are vectors of velocity and acceleration respectively. Stating second order dynamics model, $m_{i}$ is evidently mass of the vehicle.

In some previous works (De Gennaro (2006)) and (Stipanovic (2007)), first order dynamics has been used to describe the dynamical characteristics of the vehicles. Second order dynamics enables us to obtain a more realistic model 
while dealing with constraints of accelerations and velocities. The workspace of the vehicles consists of obstacles in given locations of the map. Vehicles are considered as point-mass objects with an associated visibility zone. The problem is to find the appropriate control input for every vehicle such that the one undergoing the dynamics given in (1) can reach its destination while avoiding collision with other vehicles located in its detection zone (Fig. 1). This filed is a circle with radius of visibility length unless there is an obstacle blocking the visibility. This zone emulates detection capabilities of autonomous vehicles and for comparison purposed, the field of view of a human driver. Finally, it is considered that vehicles can communicate with each other to exchange information when they are located in their respective visibility zone (Fig.1). Red vehicles are detected ones and green ones are not detected. Other vehicles will be taken into account in the navigation function provided that they are detected.

It should be pointed out that the main concern of this work is the behavior of the vehicles at the intersections so it has been

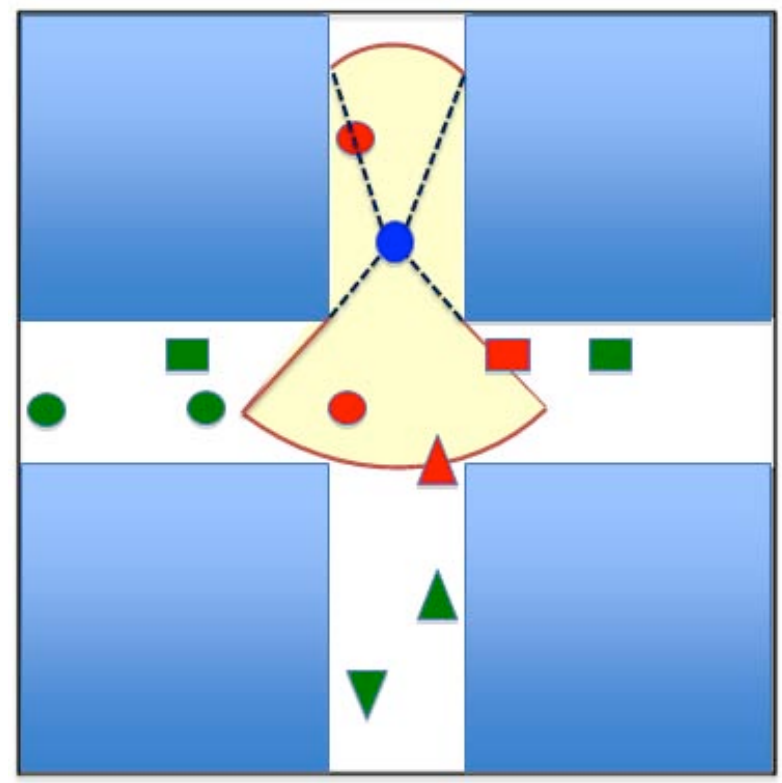

Fig. 1. Visibility zone of a given vehicle (blue). Other Vehicles could be detected if they are in its visibility zone (sectors or circle). Only these vehicles will be taken into account in the navigation function. In this example, the red vehicles are detected.

assumed that the desired destinations of the vehicles are located outside of the defined workspace. Hence, the convergence to a final configuration is not a critical issue. The main idea of the work is to take the inertia of the vehicles into account in their control system such that the vehicles with large mass (which are more sensitive to acceleration and deceleration from an energy consumption point of view) get the opportunity to leave the intersection more smoothly than the lighter ones. Inertia is the resistance to moving of vehicles; in the model of second order dynamics in (1) the resistance for moving is the mass of every vehicle.
The vehicles are considered to follow a given lane. So, no static obstacles are considered and only vehicles in the same or in crossing lanes are taken into account. The vehicles coming across in a two-way-street induced no collision risks.

\section{DECENTRALIZED NAVIGATION FUNCTION}

A navigation function is practically a smooth mapping which should be analytic in the workspace of every vehicle and its gradient would be attractive to its destination and repulsive from other vehicles. So, an appropriate navigation function could be combined with a proper control law in order to obtain a trajectory for every vehicle leading to the destination and avoiding collisions. Although the navigation functions presented by Dimarogonas (2003) and De Gennaro (2006) provide a stable solution and exhibit strong analytical properties, it has not been studied from scalability and computation point of views. In the navigation problem as formulated in this paper, the main purpose is to modify their navigation function to take into account the dynamical characteristics of the vehicles. In such a way, it is expected to get autonomous behaviors that can mimic the typical reaction of human drivers at intersection, such as breaking or accelerating when it is anticipated that, as example, a crossing truck will have not time to stop to respect priority. This actually adds the possibility of energy optimization at the intersections, by limiting as much as possible the costly velocity changes.

As a consequence, the navigation function proposed by Roozbehani (2009, a) is exploited. It is well conditioned to handle local traffic conditions in which many vehicles are involved. As it has been mentioned above, no static obstacles are present in the predefined lanes. So, the corresponding term is suppressed.

$$
\phi_{i}=\lambda_{1}\left\|q_{i}-q_{d i}\right\|^{2}+\lambda_{2} \sum_{i \neq j} V(i, j) \frac{1}{\beta_{\sigma}\left(q_{i}, q_{j}\right)}
$$

The proposed function (2) is composed of two components. The first term is the squared distance of vehicle $i$ from its destination and attains small values as the vehicle approaches the goal. The second term aims at avoiding collision between vehicle $i$ and all other vehicles located in its visibility zone. Priority coefficients $V(i, j)$ have been introduced so that the vehicle $i$ will change its speed according to the priorities given to vehicles $j$. A larger priority coefficient leads to higher acceleration or deceleration of vehicle $i . V(i, j)$ is chosen as the ratio between the inertia of vehicle $i$ and vehicle $j$. Metrics related to energy consumption or energy deficiencies could be also integrated in this matrix. Various functions can be chosen for $\beta_{\sigma}($.), providing that core properties are kept. These properties are directly connected to the visibility zone of the vehicles and the fact that the navigation function should be an analytic mapping. This function should be small when vehicle $j$ is in the visibility zone of vehicle $i$ in order to create a strong repulsive force and avoid collision risks. This function should be equal to 1 when the vehicle $j$ is out of visibility zone of vehicle $i$. The function $\beta_{\sigma}($.) given in (3) has been chosen. Its value is 
close to zero for very short distances between two vehicles and is equal to 1 at distance $\sigma$.

$$
\begin{aligned}
& \beta_{\sigma}\left(q_{i}, q_{j}\right)= \\
& \left\{\begin{array}{cc}
3\left(\frac{\left\|q_{i}-q_{j}\right\|}{\sigma}\right)^{2}-2\left(\frac{\left\|q_{i}-q_{j}\right\|}{\sigma}\right)^{3} & \text { if }\left\|q_{i}-q_{j}\right\|<\sigma \\
1 & \text { else }
\end{array}\right.
\end{aligned}
$$

According to the navigation function presented in (2) and the vehicles dynamics defined in (1), the following control law is proposed:

$$
u_{i}=-k_{i} \nabla_{q_{i}} \phi_{i}
$$

In every step, the vehicle will move according to gradient descent method. $k_{i}$ is step-size parameter that could be tuned in order to have a collision free crossing. As it has been mentioned before all vehicles move in their predefined lane. This means that vehicles do not move laterally.

\section{ANALYSIS OF THE NAVIGATION FUNCTION}

In this section we analyze the properties of the proposed navigation function, which could be used to control each vehicle to reach its desired destination. As previously mentioned, it does not guarantee the convergence of all vehicles to their destination points in the presence of other vehicles.

The objective is to identify potential deadlock situations and to investigate practical solutions to overcome them. Critical points can be identified by analyzing the gradient (5) of the navigation function introduced in (2).

$$
\begin{aligned}
& \nabla_{q_{i}} \phi_{i}=2 \lambda_{1}\left(q_{i}-q_{d i}\right) \\
& -\lambda_{2} \sum_{j \neq i} \frac{V(i, j)}{\beta_{\sigma}\left(q_{i}, q_{j}\right)} \frac{\partial \beta_{\sigma}\left(q_{i}, q_{j}\right)}{\partial q_{i}}
\end{aligned}
$$

The first term is the gradient of attractive potential to the destination and shows the distance between current position of the vehicle and its destination. This term plays an important role because it results in the shortest trajectory on the predefined lane to reach the destination in absence of other vehicles. As the main focus is the coordination of the vehicles at intersections, the destination is changed over time in order to get an invariant attractive force.

The second term corresponds to the gradient of the term associated with propulsive force from other vehicles.

The critical points of $\phi_{i}$ are obtained when the gradient is set to zero:

$$
\begin{aligned}
& \nabla_{q_{i}} \phi_{i}=0 \Rightarrow \\
& 2 \lambda_{1}\left(q_{i}-q_{d i}\right)=\lambda_{2} \sum_{j \neq i} \frac{V(i, j)}{\beta_{\sigma}\left(q_{i}, q_{j}\right)} \frac{\partial \beta_{\sigma}\left(q_{i}, q_{j}\right)}{\partial q_{i}}
\end{aligned}
$$

Two different cases are possible defining equilibrium in (6).
If $\quad\left(q_{i}-q_{d i}\right)=0 \quad$ and $\quad \sum_{j \neq i} \frac{V(i, j)}{\beta_{\sigma}\left(q_{i}, q_{j}\right)} \frac{\partial \beta_{\sigma}\left(q_{i}, q_{j}\right)}{\partial q_{i}}=0$

(case 1), the vehicle has reached its destination. Hence, from $\frac{\partial \beta_{\sigma}\left(q_{i}, q_{j}\right)}{\partial q_{i}}=0$ we can say that there is no other vehicle in its visibility zone. This case is not a critical one and just help in finding the instant of convergence to the desired destination.

$$
\text { If } \quad\left(q_{i}-q_{d i}\right) \neq 0 \quad \text { and } \quad \sum_{j \neq i} \frac{V(i, j)}{\beta_{\sigma}\left(q_{i}, q_{j}\right)} \frac{\partial \beta_{\sigma}\left(q_{i}, q_{j}\right)}{\partial q_{i}} \neq 0
$$

(case 2), the first condition implies that the $i$ th vehicle does not reach the desired destination and the second condition implies that there is at least one other vehicle in its visibility zone. The equality of these two conditions means that the repulsive force from the other vehicle is actually equal to attractive force from the destination point. Clearly, this equilibrium is not a desired one. The trick to overcome this deadlock is to continuously change the destination. Alternatively, noise can be added to the measurements, which is anyway the case in practical implementations.

\section{EVALUATION}

In this section the proposed navigation function is evaluated and compared with two other methods (i.e. intersection with traffic lights and centralized control). The effectiveness of the proposed navigation function in coordinating the crossing of four vehicles is investigated. The convergence is obtained when vehicles leave the intersection without collision. For the comparison of the proposed control scenarios with the two other methods, two different criteria are defined.

\subsection{Study of the navigation function}

First, the control of four vehicles entering to an intersection using their navigation functions given in (2) is considered

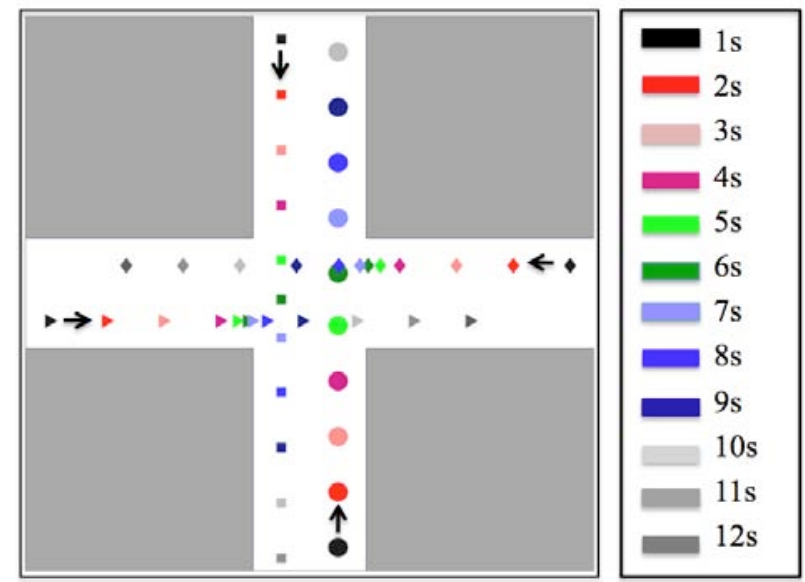

Fig. 2. Four vehicles are passing an intersection. Arrows show the direction of movement for each vehicle. The four vehicles are represented with four different shapes (circle, square, diamond, triangle). A given color corresponds to a given simulation instant listed on the left side of the figure. 
(Fig. 2). The effect of taking inertia into account to get a smoother flow for vehicles is also illustrated.

In the simulation, the four vehicles start at the same time from four different positions located at the same distance of the intersection. So, without maneuvers, they are all expected to reach the intersection at the same time. The distance from the intersection is long enough so that each vehicle can reach its maximal velocity before getting close to the intersection.

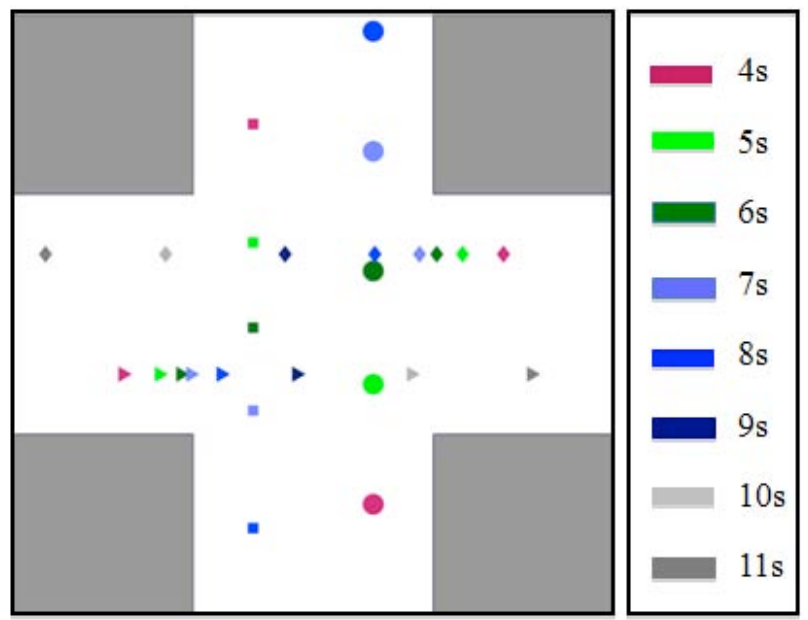

Fig. 3 shows the interval between the time vehicles enter the center of intersection and the time they successfully solve the intersection problem. This interval corresponds to the time interval between forth second and $8^{\text {th }}$ second (Fig.1).

The chosen integration step for the simulation is $20 \mathrm{~ms}$. Values of the parameters in the navigation function are $\lambda_{1}=0.1$ and $\lambda_{2}=0.035$. The value of controller gain introduced in (4) is $k_{i}=1$ for all four vehicles.

Fig.2 illustrates the motion of the vehicles by displaying their position each second. A given shape corresponds to a given vehicle and a given color corresponds to a given time. The black arrows indicate the direction of motion. After four seconds, all vehicles enter simultaneously the visibility zone of the others and their velocities start to change.

The vehicle represented with circles (Fig.2) is five times heavier than other three vehicles. Thanks to the chosen navigation function, its velocity does not change significantly. All other three vehicles have same weight, however it can be seen that the vehicle represented by squares keep also almost the same velocity. This is because the two other vehicles have anyway to decelerate to free the intersection for the heavier vehicle.

Fig. 3 shows a zoomed view of the intersection for the same simulation case. Acceleration and deceleration are visible and collisions are avoided.

Fig. 4 shows the velocities of the four vehicles before, during and after the crossing at the intersection. As mentioned previously, the velocity of the heavier vehicles does not change significantly. It is important to underline that, with

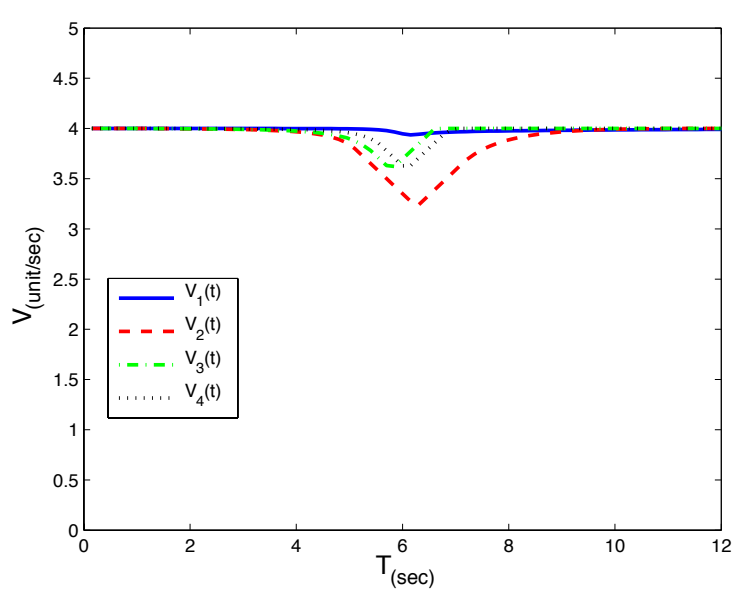

Fig. 4. Change of velocities for four vehicles passing an intersection. Heavier vehicle has lesser change in velocity than others.

the chosen navigation function parameters, none of the vehicles have to stop. As a consequence, the crossing is handle in a very smooth way.

In absence of other vehicles, each vehicle speeds up with its maximum acceleration to obtain its maximal velocity. When any other vehicle could be detected it breaks as a result of repulsive force sensed from the other vehicle. To ensure the collision avoidance it will break with its maximum absolute value of acceleration. After successfully solving the intersection problem, the vehicle will speed up again to reach its desired destination. This is the reason, that maximum acceleration is seen as the rate of change in the speed of every vehicle in Fig.3.

\subsection{Energy efficiency}

Here, the coordination of autonomous vehicles at intersections using the proposed navigation function is compared to two other extreme scenarios. First, the less effective one with drivers obeying to traffic lights. Second, the most effective one with an optimal centralized control that relies on a full knowledge of all the vehicles and their environments. The objective is to show whether a decentralized approach that relies only on local information and on limited computation power can exhibit performances close to the optimal scenario.

For comparing these three scenarios, the same four-way intersection with one lane of traffic in each direction as considered in the previous subsection (Fig.1) is simulated.

As first control scenario we simulate traffic lights. There are two traffic lights, which have been configured such that each vehicle is given a green light for 4 seconds, a yellow one for 1 second, and a red one for 4 second. Although there are qualified works concerning the timing of the traffic lights in the literature, they cannot be considered here for two main reasons. First, most of the works have been done for the management of multiple intersections, while our focus is in solving the problem at each intersection. Second, vehicles appear in each direction symmetrically, which very much simplifies the timing problem. As a consequence, a 
symmetric timing pattern is selected as mentioned before that minimizes the global energy consumption at the intersection. As second method, the proposed navigation function is considered with different vehicle inertia. $25 \%$ of the vehicles are five times heavier than the others and they are uniformly distributed in the four direction of the intersection. So, the priority matrix introduced in (2) was assumed to have values regarding to inertias of vehicles. Vehicles have been modeled using second order dynamics, so masses of vehicles are representative of their inertias respectively:

$$
V(i, j)=\frac{m_{i}}{m_{j}}
$$

$m_{i}$ and $m_{j}$ are inertias of vehicle $i$ and vehicle $j$ respectively.

The third scenario corresponds to the method proposed by Stipanovic et la (2007), which is an example of the application of centralized control approach for navigation. In their work, they assumed the second order dynamic as ours in (1). So the behavior of all vehicles modeled as a linear timeinvariant dynamic system in the following form:

$$
\dot{X}=A X+B U
$$

$X$ is the state vector in (8) which is position and velocity of all vehicles. The cost function for overall system introduced in (9).

$$
\begin{gathered}
J=\sum_{i=1}^{N} J_{i}=\int_{0}^{\infty} q(x, u) d t \\
q(x, u)=\sum_{i=1}^{N} q_{i}\left(x_{i}, u_{i}\right)
\end{gathered}
$$

As every vehicle's goal is to arrive at its destination point, which in our case is the other side of the intersection, they associated the quadratic cost function for every vehicle. The overall cost function will be the summation of cost functions for every vehicle:

$$
q_{i}=\frac{1}{2}\left[\left(x_{i}-x_{i}^{e}\right)^{T} Q\left(x_{i}-x_{i}^{e}\right)+\left(u_{i}-u_{i}^{e}\right)^{T} R\left(u_{i}-u_{i}^{e}\right)\right]
$$

$x_{i}$ and $x_{i}^{e}$ are the vectors composed of position and velocity of vehicle $i$ at its current state and goal state respectively. $u_{i}$ and $u_{i}^{e}$ also presents the current and ideal input for the vehicle respectively.

We assumed $Q$, identity matrix and $R$ as a diagonal matrix with inertias of vehicles as its elements respected to every vehicle. The control law was computed using these two matrices and collision avoidance as constraints of the problem. . It is worth mentioning that the centralized method of navigation is not computationally efficient and hence cannot be implemented in real world applications

The three methods are compared according to two different criteria. First criterion is the weighted average of energy used by the vehicles passing through the intersection. Energy consumption corresponds to control signal, i.e. acceleration and deceleration of the vehicles at the intersection. It is worth
Table 1. Comparison of three control methods for vehicles passing through an intersection by indexes as mean of energy consumption of every vehicle and maximum rate of vehicles entering the intersection.

\begin{tabular}{|l|l|l|}
\hline Control scenario & $\begin{array}{l}\text { Energy } \\
\text { criterion } \\
\text { according to } \\
(5)\end{array}$ & $\begin{array}{l}\text { Maximum } \\
\text { vehicle rate per } \\
\text { second to the } \\
\text { intersection }\end{array}$ \\
\hline Traffic lights & 45.6 & 1.4 \\
\hline $\begin{array}{l}\text { Decentralized control } \\
\text { using navigation } \\
\text { function }\end{array}$ & 14.14 & 2.4 \\
\hline Central Controller & 9.86 & 2.6 \\
\hline
\end{tabular}

mentioning that it has been considered that vehicles with more inertia are consuming more energy in acceleration and deceleration. The energy consumption index is defined as follows:

$$
\bar{E}=\frac{1}{T_{f} N} \sum_{i} J_{i}=\frac{1}{T_{f} N} \sum_{i} \int_{0}^{T_{f}} u_{i}^{T} R_{i} u_{i}
$$

In (5) $T_{f}$ is the duration of the experiment and $N$ is the total number of vehicles introduced in the system. The control signal is considered being zero when the vehicle exits the system.

The second criterion is the maximum pace at which vehicles could enter the intersection without generation queues longer than four non-moving vehicles.

For the first criterion, the vehicles are created simultaneously and symmetrically in the four directions. Each vehicle are created at the same distance of the intersection and with their maximum velocity. In such a way, their initial acceleration has no impact. The vehicle creation rate is 2 vehicles per second. This means that, every two seconds four vehicles enter the whole system from the four different directions. This generation pattern is applied for all the three control scenarios.

The creation of vehicles to compute the second criterion is slightly different. In this case, vehicles are continuously created, providing that there are not more than four vehicles in a queue to enter the intersection. So this means that every time one vehicle could pass the intersection successfully, one new vehicle is created in the same direction of the previous one. This vehicle is created in a distance from the intersection similar to the previous vehicle. By this method of creating the vehicle, we compute the total number of vehicles that could enter the whole system in 600 seconds and calculate the rate of vehicles entering the intersection per second.

Table. 1 shows the comparison of these three different scenarios according to the two different criteria.

As it is clear from this table that the proposed navigation function induces energy consumption between the two other methods. The proposed method is only $30 \%$ less effective than the centralized one, while being easily implementable. 


\section{CONCLUSION}

In this work the previous decentralized navigation function (Roozbehani 2009) has been modified in two ways. First, the avoidance force from other vehicles is only active in the bounded sensing regions of each individual vehicle. Second, the navigation function has been modified in a way to optimize energy consumption taking the inertia of vehicles into account. This paves the way towards an on-board energy optimization by indirectly giving priority to heavier vehicles at intersections. The proposed method has been compared with the two extreme approaches of centralized control and traffic lights. The proposed method shows a significant improvement in comparison with the classic traffic lights from an energy point of view and also the rate of vehicles an intersection can simultaneously and smoothly handles.

Our future research directions include the analytical study of the convergence of the proposed coordination approach. More realistic features will be added, like turns and multiple lanes in the same direction. In future we will also study the behavior of the vehicles under communication constraints and lack of energy as it could happen when using electrical vehicles.

\section{REFERENCES}

Baras, J. S., Tan,X. and Hovareshti, P. (2003). Decentralized control of autonomous vehicles. Pages 1532-1537. Proceeding of IEEE Conference on Decision and Control, Maui, Hawaii.

Beard, R.W., McLain,T.W. (2003) Multiple UAV cooperative search under collision avoidance and limited range communication constraints. Page 2530- 2003. Proceeding of IEEE Conference on Decision and Control, Maui, Hawaii.

De Gennaro, M. C., Jadbabaie A., (2006) Formation control for a cooperative multi-agent system using decentralized navigation functions. Page 1346-1351. Proceedings of the American Control Conference. Minneapolis, Minnesota, USA.

Dimarogonas, D. V., Zavlanos, M. M., Loizou, S. G. Kyriakopoulos, K. J. (2003) Decentralized motion control of multiple holonomic agents under input constraints. Page 3390-3395. Proceeding of IEEE Conference on Decision and Control, Maui, Hawaii.

Prandini,M., Sastry,S. (2004) Optimal coordinated motions of multiple agents moving on a plane. SIAM Journal on Control and Optimization, 42(2), pages 637-668.

Roozbehani, H., Rudaz, S., Gillet, D. (2009) A HamiltonJacobi formulation for cooperative control of multi-agent systems. IEEE International Conference on Systems, Man and Cybernetics, San Antonio, Texas, USA.

Roozbehani, H., Rudaz, S., Gillet, D. (2009) On decentralized navigation schemes for coordination of multi-agent dynamical systems. IEEE International Conference on Systems, Man and Cybernetics, San Antonio, Texas, USA.

Stipanovic, D.M., Hokayem, P. F., Spong, M.W. Siljak.D.D. (2007) Cooperative avoidance control for multi-agent systems. Journal of Dynamic Systems, Measurement, and Control, Pages 129-699. 